\title{
Special Section Guest Editorial: Selected Papers from the 3rd Mediterranean Conference on Nanophotonics
}

\author{
Zeev Zalevsky, ${ }^{a}$ Ekmel Ozbay, ${ }^{\text {b }}$ and Ibrahim Abdulhalim ${ }^{c}$ \\ ${ }^{a}$ Bar-Ilan University, School of Engineering, Ramat-Gan 52900, Israel \\ zalvesz@biu.ac.il \\ ${ }^{\mathrm{b}}$ Bilkent University, Nanotechnology Research Center (NANOTAM), Bilkent, \\ Ankara 06800, Turkey \\ ozbay@bilkent.edu.tr \\ ${ }^{\mathrm{c}}$ Ben-Gurion University, Electro-optics Engineering, Beer-Sheva 84105, Israel \\ abdulhlm@bgu.ac.il
}

The 3rd Mediterranean Conference on Nanophotonics (MediNano-3) was held in Belgrade, Serbia, on 18-19 October 2010. More than 150 participants from many Mediterranean as well as non-Mediterranean countries participated in the meeting. In total, 95 contributions were presented at the meeting, including 2 plenary, 4 keynote, and 30 invited papers. Of the submitted contributions, 59 were accepted, including 15 for oral presentations.

As in the previous MediNano meetings, the purpose was to promote collaboration within the field of nanophotonics among scientists, research facilities, and institutes from Mediterranean countries and to establish a tradition of future nanophotonics conferences.

The conference also targeted young scientists, and indeed the topics presented in the meeting attracted a large number of students who submitted their papers.

A large number of manuscripts were submitted for this special section focusing on nanophotonic devices, metamaterials, and fabrication, as well as characterization techniques.

The fourth MediNano meeting will be held at the Universita' di Roma La Sapienza, Rome, Italy, on 24-25 October 2011.

$1934-2608 / 2011 / \$ 25.00$ @ 2011 SPIE 